\title{
Anthropometric Evaluation of a 3D Scanning Mobile Application
}

\author{
Brooke E. SMITH *, Marcelline DECHENAUD, Steven B. HEYMSFIELD \\ LSU Pennington Biomedical Research Center, Baton Rouge LA, USA
}

https://doi.org/10.15221/21.33

Key Words: 3D body scanning, mobile application, 3D optical

\section{Introduction}

Anthropometric measurements have long been used to study physical human variation. Throughout history, and beginning in the late 1800s, anthropometry has been used in many ways including: human identification [1], correlations to physical traits, and associations with underlying medical problems and diseases [2,7], socioeconomic status [3], and more.

Since anthropometry has such versatile uses, the need for more advanced, non-contact-based measures of anthropometry became apparent. This was accomplished in the late 1900s by Loughborough University [4], and the field would become known as digital anthropometry. Moving forward to today, 3D body scanning has been increasingly used in clinical and research settings for physical human evaluation. However, these new, high-tech devices tend to be costly and difficult to transport.

Because of the limited accessibility, high cost, and low portability of these devices, a more accessible means to the uses of digital anthropometry was needed. Smartphone applications have been developed in recent years that have similar capabilities to these large and expensive devices. The current study aimed to compare conventional body circumferences at standard locations [5] to those generated digitally by a smartphone application (MeThreeSixty; SizeStream LLC, Cary, NC)) and a professional grade imaging system (SizeStream SS20; Cary, NC). Digital outputs (.obj files) from both scanners were acquired from a sample of healthy adults and processed with universal software (US) developed in our laboratory [6]. The device-agnostic US was developed to standardize and measure anthropometric dimensions (circumferences, lengths, surface areas, and volumes) from 3D body scans, using identical anatomic landmark definitions across devices and methods [6].

\section{Methods}

Participants were 10 healthy adults ([mean \pm SD] age $41.8 \pm 22.4$ years; BMl $27.7 \pm 5.8 \mathrm{~kg} / \mathrm{m}^{2}$ ). Participants were all measured and recruited to LSU Pennington Biomedical Research Center.

Conventional standardized anthropometric measurements were made with a flexible tape measure at the waist, hips, right and left mid-upper arm, and right and left mid-thigh outlined by the US National Health and Nutrition Examination Survey. Measurments made with a flexible tape measure were made in triplicate to the nearest $0.1 \mathrm{~cm}$. Digital anthropometric scans were performed using the MeThreeSixty application and the SizeStream SS20 in each participant. Duplicate scans were taken for both MeThreeSixty and SizeStream SS20. Acquired 3D .obj files were processed using the US. The image processing software is run in MATLAB and produced standardized circumference measurements.

After US standardization, the digital measurements acquired from the SizeStream SS20 and MeThreeSixty application were compared to the corresponding flexible tape reference measurements. Statistical analyses were generated in Microsoft Excel 2021, and tables were created in Microsoft Word 2021. Of the total participant sample, all 10 were evaluated on the SS20 and on the MeThreeSixty application. The anonymized data set necessary to replicate the current study findings can be obtained by request from the authors. To test the agreement between the SizeStream SS20 and MeThreeSixty measurements derived from universal software to their matching conventional anthropometric measurements, absolute error and root mean square errors were calculated.

\section{Results}

Overall, circumferences measured with the app-US approach agreed closely with those acquired with the flexible tape as shown in Table 1 (absolute errors, $0.79-1.96 \mathrm{~cm}$; root-mean square errors, 1.32 $3.97 \mathrm{~cm}$ ). Results were largely equivalent to those from the SizeStream SS20 scanner (Table 2; mean absolute errors, 0.71-5.6 cm; RMSE, 2.00-11.75 cm). 
Table 1. Mean $\pm S D$, Mean Absolute Error, and RMSE of conventional anthropometry measurements vs. MeThreeSixty application measurements. Application measurements standardized by US.

\begin{tabular}{|c|c|c|c|c|}
\hline Units: cm \pm SD & Anthro & App & Mean Abs. Error & RMSE \\
\hline Waist & $91.91 \pm 14.72$ & $92.38 \pm 13.78$ & $1.88 \pm 2.12$ & 3.59 \\
\hline Hip & $107.48 \pm 13.53$ & $108.37 \pm 11.93$ & $1.38 \pm 3.09$ & 3.97 \\
\hline Arm (L) & $34.54 \pm 5.33$ & $32.14 \pm 4.68$ & $1.96 \pm 1.43$ & 2.88 \\
\hline Arm (R) & $34.57 \pm 5.34$ & $32.51 \pm 4.63$ & $1.75 \pm 1.31$ & 2.51 \\
\hline Thigh (L) & $59.42 \pm 7.40$ & $58.97 \pm 7.88$ & $0.79 \pm 0.75$ & 1.32 \\
\hline Thigh (R) & $59.82 \pm 7.44$ & $58.97 \pm 8.09$ & $1.08 \pm 1.36$ & 2.09 \\
\hline
\end{tabular}

Table 2. Mean $\pm S D$, Mean Absolute Error, and RMSE of conventional anthropometry measurements vs. the SizeStream SS20 scanner measurements. SS20 measurements standardized by US.

\begin{tabular}{|l|c|c|c|c|}
\hline Units: $\mathbf{c m} \pm$ SD & Anthro & SS20 & Mean Abs. Error & RMSE \\
\hline Waist & $91.91 \pm 14.72$ & $101.53 \pm 18.85$ & $5.6 \pm 7.10$ & 11.75 \\
\hline Hip & $107.48 \pm 13.53$ & $109.82 \pm 12.90$ & $1.67 \pm 3.25$ & 4.10 \\
\hline Arm (L) & $34.54 \pm 5.33$ & $35.88 \pm 5.02$ & $1.24 \pm 1.13$ & 2.00 \\
\hline Arm (R) & $34.57 \pm 5.34$ & $36.16 \pm 8.43$ & $0.71 \pm 4.16$ & 4.57 \\
\hline Thigh (L) & $59.42 \pm 7.40$ & $58.64 \pm 8.52$ & $2.06 \pm 1.07$ & 2.53 \\
\hline Thigh (R) & $59.82 \pm 7.44$ & $59.17 \pm 9.19$ & $1.80 \pm 1.57$ & 2.81 \\
\hline
\end{tabular}

\section{Discussion}

These initial results suggest that the MeThreeSixty application's measurements are comparable to those made by conventional anthropometry. The current study presents a new approach to obtaining digital circumferences and addresses the need for a widely available, comparable means to obtaining body measurements outside of a research facility with a highly trained staff expert in anthropometric measurements. The universal software approach used in the study allowed the researcher to standardize measurements across devices and gain an informed opinion on the agreement between the two devices and conventional anthropometry by a trained staff member. The smartphone application approach has the potential to allow participants (or consumers) to gain access to accurate measurements, and therefore some cardiovascular and health risk assessments [2,7], from the comfort of their own home. This application is now free to download on the app store, providing consumers with a method comparable to expensive professional grade scanners from their home environment. A larger sample to follow up on these pilot evaluations is now being collected.

\section{Conclusions}

This initial evaluation of the MeThreeSixty app opens up the possibility of acquiring digital standardized anthropometric measurements that can be used to derive estimates of body fat, other clinically relevant compartments, and health risk predictions at little to no cost. The app's accuracy is comparable to conventional flexible tape-anthropometry administered by a trained technician and similar to data acquired by a professional-grade 3D optical scanner. The app's accessibility makes it useful for monitoring and tracking body measurements in settings outside of specialized facilities.

\section{Acknowledgement}

The authors would like to thank SizeStream for providing the SS20 scanner, and technical assistance with the MeThreeSixty application. Additionally, the research would have not been possible without the facilities available to us at Pennington Biomedical Research Center. 


\section{References}

[1] Kewal Krishan: Anthropometry in Forensic Medicine and Forensic Science-'Forensic Anthropometry': The Internet Journal of Forensic Science. 2007; Volume 2, Number 1.

[2] Olds, Tim, et al. "12-Anthropometry, Health, and Body Composition." Anthropometrica: A Textbook of Body Measurement for Sports and Health Courses, edited by Kevin Norton, UNSW Press, 2004, pp. 366-385.

[3] Bogin B, Keep R. Eight thousand years of economic and political history in Latin America revealed by anthropometry. Ann Hum Biol. 1999 Jul-Aug;26(4):333-51. doi: 10.1080/030144699282651. PMID: 10462154.

[4] Heymsfield, Steven B et al. "Digital anthropometry: a critical review." European journal of clinical nutrition vol. 72,5 (2018): 680-687. doi:10.1038/s41430-018-0145-7

[5] Centers for Disease Control and Prevention (CDC ), National Center for Health Statistics (NCHS). National Health and Nutrition Examination Survey (NHANES): Anthropometry Procedures Manual. Hyattsville, MD: Centers for Disease Control and Prevention; 2007

[6] Sobhiyeh S, Kennedy S, Dunkel A, et al. Digital anthropometry for body circumference measurements: Toward the development of universal three-dimensional optical system analysis software. Obes Sci Pract. 2021;7:35-44. https://doi.org/10.1002/osp4.467

[7] Goh LGH, Dhaliwal SS, Welborn TA, et al. Anthropometric measurements of general and central obesity and the prediction of cardiovascular disease risk in women: a cross-sectional study. BMJ Open 2014;4:e004138. doi:10.1136/bmjopen-2013- 004138 